\title{
A spectral study of Vega: A rapidly-rotating pole-on star
}

\author{
Graham Hill $^{1,4}$, Austin F. Gulliver ${ }^{2,4}$ and Saul J. Adelman ${ }^{3,4}$ \\ ${ }^{1}$ Department of Physics, University of Auckland, Auckland, New Zealand 1001 \\ email: gp-hill@xtra.co.nz
}
${ }^{2}$ Department of Physics and Astronomy, Brandon University, Brandon, MB R7A 6A9, Canada email: gulliver@brandonu.ca
${ }^{3}$ Department of Physics, The Citadel, 171 Moultrie Street, Charleston, SC 29409, USA email: adelmans@citadel.edu

${ }^{4}$ Guest Investigator, Dominion Astrophysical Observatory, Herzberg Institute of Astrophysics, National Research Council of Canada, 5071 W. Saanich Road, Victoria, BC V9E 2E7, Canada

\begin{abstract}
Ultra-high signal-to-noise, high dispersion spectroscopy over the wavelength range $\lambda 4487-4553$ shows Vega to be a rapidly rotating star $\left(V_{\text {eq }} \sim 160 \mathrm{~km} \mathrm{~s}^{-1}\right)$ seen almost pole-on. These data, analyzed anew, are combined with analyses of the hydrogen lines $(\mathrm{H} \gamma, \mathrm{H} \beta$ and $\mathrm{H} \alpha)$ and the latest absolute continuum flux for Vega to yield the following results: $V \sin i=21.9 \pm$ $0.1 \mathrm{~km} \mathrm{~s}^{-1}$, polar $T_{\text {eff }}=9680 \pm 10 \mathrm{~K}$, polar $\log g=4.00 \pm 0.02 \mathrm{dex}, V_{\text {eq }}=160 \pm 10 \mathrm{~km} \mathrm{~s}^{-1}, \xi_{\mathrm{T}}=$ $1.08 \pm 0.02 \mathrm{~km} \mathrm{~s}^{-1}$ and $i=7.9 \pm 0.5^{\circ}$. The variations in $T_{\text {eff }}$ and $\log g$ over the photosphere total $350 \mathrm{~K}$ and $0.06 \mathrm{dex}$, respectively. The mean $T_{\text {eff }}=9510 \pm 10 \mathrm{~K}$ and mean $\log g=3.97 \pm 0.02 \mathrm{dex}$ agree with the spherical model values derived here and by others.
\end{abstract}

Keywords. Line: profiles, techniques: spectroscopic, stars: fundamental parameters, stars: individual (Vega), stars: rotation

\section{Introduction}

Since Johnson \& Morgan (1953) included Vega $(\alpha$ Lyrae $=$ HD $172167=$ HR 7001) as one of the ten primary UBV standards the star has been used extensively as a primary and secondary spectrophotometric standard in the near infrared, optical, and ultraviolet regions (Hayes 1985, Bohlin et al. 1990). Studies of its elemental abundances show that it is metal-weak by [-0.6] dex (Adelman \& Gulliver 1990) compared to the Sun.

Castelli \& Kurucz (1994) compared blanketed LTE models for Vega calculated with the new ATLAS9 and ATLAS12 codes (Kurucz 1993) with the observed energy distribution and Balmer line profiles. Their preferred model had $T_{\text {eff }}=9550 \mathrm{~K}, \log g=3.95 \mathrm{dex}$ and a microturbulent velocity $\xi=2 \mathrm{~km} \mathrm{~s}^{-1}$ for a metallicity $[\mathrm{M} / \mathrm{H}]=-0.5$.

Aumann et al. (1984) found that Vega is surrounded by a dust shell or disk of diameter about 30 arcsec. Recent research has concentrated on imaging Vega in the IR and near IR. Van der Bliek et al. (1994) measured diameters of $22 \pm 2$ and $35 \pm 5$ arcsec. The latter value was confirmed by Heinrichsen, Walker \& Klaas (1998) who found $36 \pm 3$ arcsec. Hanbury Brown et al. (1974) measured a limb-darkened angular diameter of $3.24 \pm 0.07$ arcsec, a result confirmed by Ciardi et al. (2001) who obtained $3.28 \pm 0.01$ arcsec. These results are not without uncertainty since they depend on a limb darkened model for interpretation, but they lead to a radius of $2.73 R_{\odot}$.

Vega's weak line profiles as seen in high $S / N$ spectra are clearly flat-bottomed resulting in a trapezoidal appearance while the strong lines exhibit normal rotationally-broadened profiles (Gulliver et al. 1994). Gray $(1985,1988)$ suggested that Vega might be a star 
seen pole-on because of its excessive luminosity. The program STELLAR was developed to demonstrate that a rotating model with a temperature gradient over the photosphere could produce flat-bottomed profiles and found that Vega could be modelled as a pole-on star seen at an angle $i=5^{\circ}$. Our present work, based in part on a criticism by Peterson (2003, private communication), shows that $i$ is closer to $8^{\circ}$.

Various techniques have been developed to determine the fundamental parameters of stars from observational data. These have included photometric calibrations; Balmer line matching and continuous flux matching to obtain $T_{\text {eff }}$ and $\log g$; fine analysis to obtain abundances and $\xi_{\mathrm{T}}$, spectral line fitting to obtain $V \sin i$ and Doppler imaging to obtain the inclination of the rotational axis. Given the proper conditions the program STELLAR is capable of determining all of these parameters for a star by performing a grand simultaneous fit to the continuous flux, the Balmer lines and the line spectrum. In practice, however, combining all the data in such a way results in a serious loss of weight for the parameters because no data set is synthesized accurately as judged by the fit of the model to the various observational data. Generally we treat each data set independently, fixing parameters for some data where the dependency is weak (such as $T_{\text {eff }}$ in a hydrogen profile) and solving for others.

\section{Observations}

Vega was observed with the Dominion Astrophysical Observatory 1.2-m telescope using the coudé spectrograph and a 1872 pixel bare Reticon with $15 \mu$ pixels and more recently with a variety of $\mathrm{CCDs}(2 \mathrm{~K}$ and $4 \mathrm{~K}$ format). The portion of the spectrum of Vega discussed here extends from $\lambda 4487-4553$ in wavelength steps of $0.035 \AA$ and has a mean $S / N=3300$ for the continuum regions after the co-addition of two spectra. Digitized instrumental profiles of the spectrograph plus detector were constructed by co-adding intensity-weighted lines from the comparison spectra. The resultant FWHM for the instrumental profile was $0.080 \AA$. The comparison star, o Peg, used to correct the laboratory gf values was treated similarly and had a mean $S / N=840$.

The continuous energy calibration of Vega is a combination of the ultraviolet fluxes from IUE (see Bohlin et al. 2001) combined with the Hayes (1985) visual-near IR mean energy distribution. The UV data are updated in the web site

ftp.stsci.edu/instruments/cdbs/cdbs2/calspec.html

under the name alpha_lyr_stis_001.fits and are sampled at steps of 1.2 and $1.8 \AA$ respectively for the short wavelength ranges $(\lambda 1149-2000$ and $\lambda 2000-3300)$ and with a resolution of about $6 \AA$ for the long wavelength region. We used a revised version of these data (Bohlin et al. 2001).

Profiles of $\mathrm{H} \gamma, \mathrm{H} \beta$ and $\mathrm{H} \alpha$ were taken from Peterson (1969). Unfortunately these profiles only extended $40 \AA$ from each line center and in A stars the wings of these lines have yet to reach continuum levels even $40 \AA$ from line center. We had to deal with this observational limitation by including the height and slope of the continuum as unknowns.

\section{Modelling}

We modelled Vega with the program STELLAR which is in turn based on the eclipsing binary modelling code for LIGHT2 (Hill 1979). As the first step in the modelling process, a grid of ATLAS9 models (Kurucz 1993) was calculated. This grid includes the range of stellar parameters relevant for Vega, $T_{\text {eff }}$ in steps of $500 \mathrm{~K}, \log g$ in steps of 0.25 , a metallicity of $[\mathrm{M} / \mathrm{H}]=-0.5$, and $\xi_{\mathrm{T}}=0,1$ and $2 \mathrm{kms}^{-1}$. These models used the 


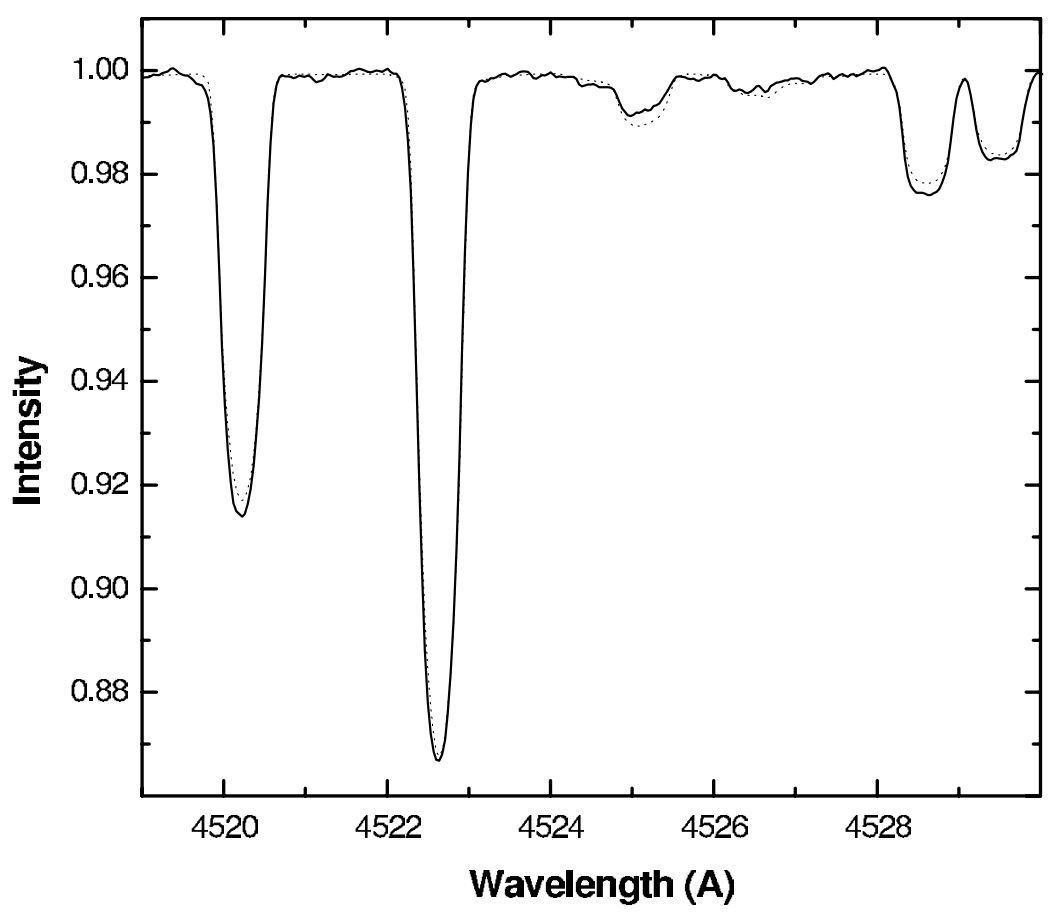

Figure 1. Vega spectrum showing the final synthetic spectrum (dashed line) and the observed line profiles (solid line) for the $\lambda 4519-4530$ region. The model parameters are $V \sin i=21.9 \mathrm{~km} \mathrm{~s}^{-1}$, polar $T_{\mathrm{eff}}=9680 \mathrm{~K}$, polar $\log g=4.00 \mathrm{dex}, V_{\mathrm{eq}}=160 \mathrm{~km} \mathrm{~s}^{-1}$, $\xi_{\mathrm{T}}=1.08 \mathrm{~km} \mathrm{~s}^{-1}$ and $i=7.9^{\circ}$.

improved continuum opacities, increased line opacities and finer sampling of the latest opacity distribution functions of Kurucz (1993).

As a given stellar application is addressed, input files of synthetic specific intensity line spectra are calculated using the SYNTHE program (Kurucz \& Avrett 1981) for the 17 values of $\mu=1.0$ to 0.01 normally used in ATLAS9 and for each $T_{\text {eff }}, \log g$, $\xi_{\mathrm{T}}$ and $[\mathrm{M} / \mathrm{H}]$ point in the above grid. These are then sampled every $0.01 \AA$ for the relevant wavelength intervals. The wavelengths of interest for Vega are $\lambda 4480-4580$ for the metallic line spectra and $250 \AA$ intervals centered on $\mathrm{H} \gamma, \mathrm{H} \beta$ and $\mathrm{H} \alpha$ for the hydrogen line profiles. Continuum flux files were extracted using ATLAS9 for fitting to Vega's observed continuous flux distribution.

The line parameters used were those provided with SYNTHE supplemented by astrophysical $g f$ values determined from $o$ Peg for the $\lambda 4519-4540$ interval. A portion of the high resolution spectral region we are analyzing is shown in Figure 1. Anticipating our later results we have included the synthetic spectrum as well as the observed data. The discrepancies between the observed and synthetic spectra arise from individual abundance errors as well as errors in the $\log g f$ values. Although the model could have produced a better fit by allowing $T_{\text {eff }}$ and $\log g$ to be free parameters this was not viewed as being appropriate.

Independent astrophysical gf values were determined for 31 lines in the $\lambda 4519-4540$ region using the narrow-lined ( $V \sin i=6.6 \mathrm{~km} \mathrm{~s}^{-1}$ ), A1 IV slightly metal-rich star, $o$ Peg, assuming $T_{\text {eff }}=9600 \mathrm{~K}, \log g=3.60, \xi_{\mathrm{T}}=1.3 \mathrm{~km} \mathrm{~s}^{-1}$ and the appropriate abundances (Adelman 1988). In light of new values for the stellar parameters of $o$ Peg, $T_{\text {eff }}=9500 \mathrm{~K}$, $\log g=3.75$ (Adelman et al. 2004), this analysis could be redone. 
For the analysis of the continuous flux, separate input files of continuum specific intensity were generated by ATLAS9 at the standard values of $\mu$ and at 1141 wavelengths from $506 \AA$ - $160 \mu$ for the grid of models are used. All input files, the continuous flux, the Balmer lines and the line spectra, are convolved with the appropriate instrumental profile. Because it has proven impossible to match the hydrogen profiles in the published continuous energy distribution, we removed the data in the vicinity of the Balmer and Paschen series confluences and for other individual hydrogen lines.

The physical model generates the run of temperature and gravity over the surface of a star with these values defined initially at the pole and changing as a function of rotational velocity and a gravity brightening exponent given as 0.25 for a radiative atmosphere. The rotating star is then viewed at some inclination angle. The integrations are performed using Gauss-Legendre quadrature with typically 32 by 32 integration points in the $\theta$ and $\phi$ axes, respectively. Thus we find intensity as a function of wavelength for each surface integration point $T_{\text {eff }}, \log g$ and $\mu$ are calculated by successive parabolic interpolations in the database within $\lambda, T_{\text {eff }}, \log g, \mu, \xi_{\mathrm{T}}$ and $[\mathrm{M} / \mathrm{H}]$. By summing these weighted intensities we generated a theoretical spectrum that could be compared with observation.

Obviously, depending on our needs, some of these variables are held constant. For example in our analysis of Vega we fixed the abundance at -0.5 solar. In addition to the models we incorporated results of parallax measurements from Hipparcos (ESA 1997) and interferometry (Ciardi et al. 2001) to aid us in completely specifying the system in terms of mass, radius, temperature and luminosity.

\section{Results}

Our approach to solve for Vega's stellar parameters uses the continuous energy flux to give a temperature and the hydrogen lines to determine the gravity. By solving these data jointly we arrived at polar values of $T_{\text {eff }}$ and $\log g$. These were used in the high resolution analysis to yield $V \sin i, \xi_{\mathrm{T}}$ and $i$. Then the whole process was iterated again.

\subsection{Effective temperature and surface gravity}

Naturally we tried to see if the continuous data would yield a value of $i$ independent of the line profile data. In this we were disappointed though ironically the earlier calibration of continuous flux (Bohlin et al. 1990) was successful (Gulliver et al. 1994). The observed continuum data we used were converted to $\mathrm{H}_{\nu}$ but left as vacuum wavelengths for comparison with the ATLAS9 output. We edited the hydrogen lines as noted earlier and tried to match the resolution of the observational data to the output from ALTLAS9. ATLAS9 used 'boxcar' smoothing and we matched this in STELLAR.

Test solutions of various combinations of the hydrogen profiles and continuous data revealed that the best approach was to combine these data sets. This produced excellent fits to both sets of data (see Figures 2 and 3) in which the fits are almost indistinguishable from the data (see also Table 1). A close examination of the UV calibration near $3200 \AA$ (see Figure 3 ) shows poor continuity between the observations of Hayes and the data at shorter $\lambda$ but we have chosen to accept the revised calibration at face value.

\section{2. $V \sin i$, microturbulence and inclination}

The original solution for the two flat-bottomed lines given by Gulliver et al. (1994) yielded parameter values for the polar $T_{\text {eff }}$, polar $\log g, V \sin i$, and $i$, of $9695 \mathrm{~K}, 3.75,21.8 \mathrm{~km} \mathrm{~s}^{-1}$ and $5.1^{\circ}$, respectively. The rms error of $5.0 \times 10^{-4}$ approximates a $S / N \sim 2000$, close to that of the observations themselves. Clearly a reasonable solution had been reached but a subsequent analysis of the hydrogen line and continuum data showed that the 


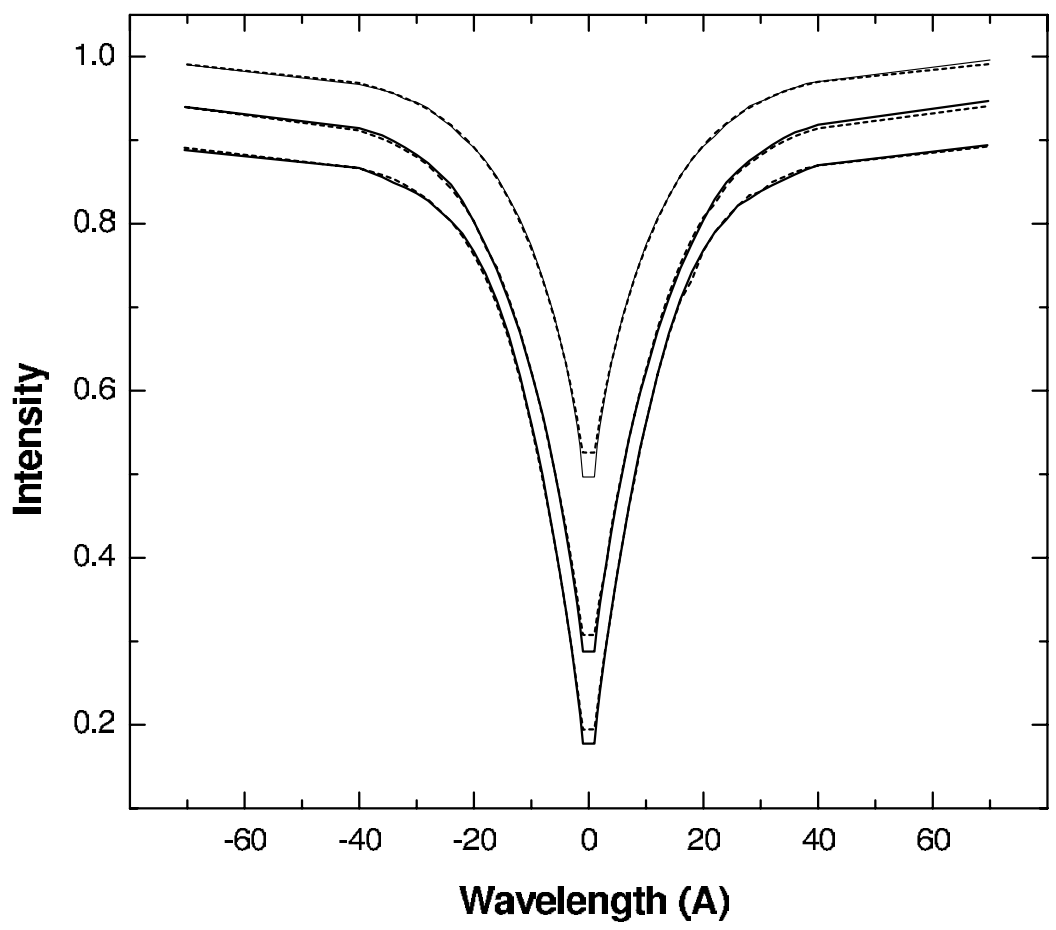

Figure 2. STELLAR model fit (dashed line) to the observed $\mathrm{H} \alpha, \mathrm{H} \beta$, and $\mathrm{H} \gamma$ hydrogen line profiles (solid lines) from top to bottom, respectively. The values of $i, V \sin i$ and $\xi$ were fixed at the values in Figure 1; only polar $T_{\text {eff }}$ and polar $\log g$ were free to vary.

Table 1. A Summary of Results

\begin{tabular}{ccclllllll}
\hline \multicolumn{9}{c}{ Mean Values } & \multicolumn{9}{c}{ Polar Values } \\
Sln & $<T><\log g>T$ & $\log g$ & $\omega / \omega_{c}$ & $i$ & $V \sin i$ & $\xi_{\mathrm{T}}$ & $\mathrm{rms}^{2}$ \\
\hline 1 & 9424 & 3.91 & $9602 \pm 35$ & $3.94 \pm .02 .48 \pm .06$ & 7.7 & $21.9 \pm .2$ & $1.08 \pm .02$ & $1.33 \mathrm{e}-6$ \\
2 & 9424 & 3.91 & 9602 & 3.94 & $.47 \pm .01$ & 7.9 & $21.9 \pm .1$ & 1.08 & $1.32 \mathrm{e}-6$ \\
3 & 9506 & 3.97 & 9680 & 4.00 & $.56 \pm .01$ & 6.4 & $21.5 \pm .1$ & $1.11 \pm .01$ & $1.37 \mathrm{e}-5$ \\
4 & 9506 & 3.97 & $9680 \pm 3$ & $4.00 \pm .01$ & & 7.8 & 21.9 & 1.08 & $8.11 \mathrm{e}-4$ \\
5 & 9506 & 3.96 & 9680 & $3.99 \pm .01$ & & 7.8 & 22.0 & 1.08 & $3.60 \mathrm{e}-5$ \\
6 & 9506 & 3.97 & $9680 \pm 4$ & 4.00 & & 7.8 & 21.8 & 1.08 & $7.63 \mathrm{e}-5$ \\
\hline
\end{tabular}

Analysis/Source: Sln 1 - Fe+Ti lines/R; Sln 2 - ג4528.29/R; Sln 3 - Fe+Ti lines/R; Sln 4 - H \& continuum/P,H,B; Sln 5 - H lines/P; Sln 6 - continuum/H,B

B - Bohlin et al. (2001)

H - Hayes (1985)

P - Peterson (1969)

$\mathrm{R}$ - Reticon

value of polar $\log g$ was too low. Peterson (2003) observed that our rotational velocity of $250 \mathrm{~km} \mathrm{~s}^{-1}$ implied a rotational breakup fraction $\omega / \omega_{\text {crit }}$ of $\sim 0.75$ as opposed to our breakup fraction $\sim 0.5$. This has a profound effect on the run of temperature and $\log g$ over the star since for the Roche model, $V_{\text {crit }}=G M / R_{\text {eq }}^{2}$ where $R_{\text {eq }}$ is the equatorial radius at breakup. For a given polar radius $R_{\mathrm{p}}, R_{\mathrm{eq}}=1.5 R_{\mathrm{p}}$ at breakup and using radii from Ciardi et al. (2001) we have $R_{\mathrm{p}} \sim 2.62 R_{\odot}$ and a mass of $M \sim 2.3 M_{\odot}$ calculated from $M=g R^{2} / G$ where $g$ is the surface gravity and $G$ the gravitational constant. These values imply a $V_{\text {crit }} \sim 340 \mathrm{~km} \mathrm{~s}^{-1}$ as opposed to the value of $500 \mathrm{~km} \mathrm{~s}^{-1}$ we had 


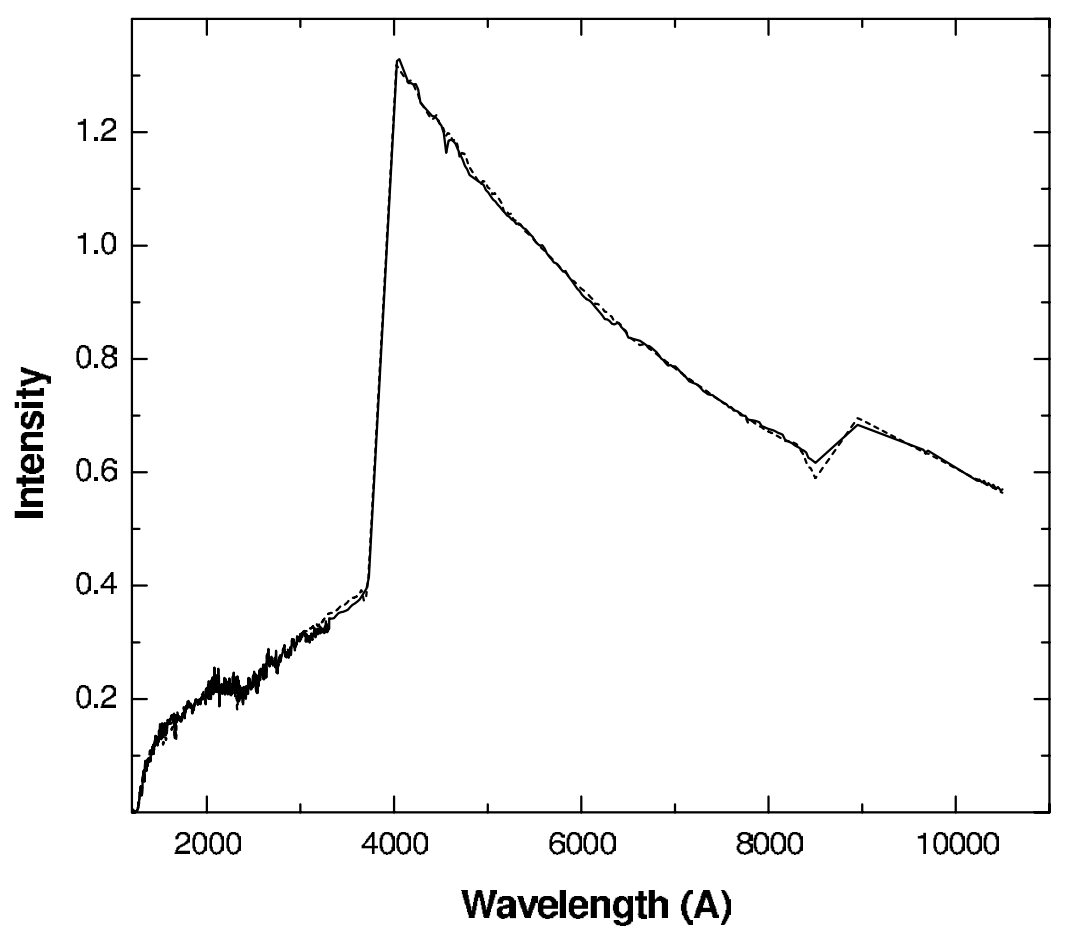

Figure 3. STELLAR model fit (dashed line) to the ultraviolet IUE fluxes of Bohlin et al. (1990) and the visual fluxes of Hayes (1985) (solid line). The Balmer and Paschen lines have been removed throughout because of incompatibilities. The values of $i, V \sin i$ and $\xi$ were fixed at the values in Figure 1; only polar $T_{\text {eff }}$ and polar $\log g$ were free to vary.

erroneously adopted. These differing values imply a value of $i$ near $8^{\circ}$ rather than the previously determined $5^{\circ}$ quoted by Gulliver et al. (1994). In addition the earlier study yielded a polar $\log g$ near 3.75 dex considerably at variance with the canonical value of 3.95 dex. Correcting for $V_{\text {crit }}$ produced consistent $\log g$ 's for all our varied data. We are grateful to Peterson for pointing out this error.

The solution proceeded iteratively since the interferometry $(\Theta=3.28$ mas $)$ and parallax $(\pi=0.1289)$ yielded $R=2.73 \pm 0.01 R_{\odot}$. Our original solution implied $\omega / \omega_{\text {crit }} \sim 0.45$ and an equatorial radius of $\sim 2.62 R_{\odot}$. Given the polar radius and the value of $\log \mathrm{g}$ $\sim 3.94$ dex our solutions predicted a mass $\sim 2.42 M_{\odot}$ and hence a breakup velocity of $340 \mathrm{~km} \mathrm{~s}^{-1}$. This value was used throughout our analysis. Rather than solve for inclination we solved for the breakup fraction $\omega / \omega_{\text {crit }}$ and derived $i$ from that. The rms of the fit implies a $S / N \sim 1000$ but the results are better than that since the presence of unidentified lines are biasing this result. The results are shown in Table 1. Note that in Table 1, if an error is quoted the associated variable was allowed to be free in the fit and if no error is present the variable was fixed. In the last two columns of the table the nature of the analysis and the data fitted are shown. The final set of stellar parameters are an amalgamation of the six solutions given.

The best values of $T_{\text {eff }}$ and $\log g$ from the continuum and hydrogen line results were adopted and for all subsequent solutions these polar values were fixed at to $9680 \mathrm{~K}$ and 4.00, respectively. Having fixed these values we concentrated on the two flat bottomed lines and solved for $V \sin i$ and $i$ after determining the microturbulence from the fit to the $\lambda 4519-4530$ region of Figure 1 . Note that microturbulence is a minor component 


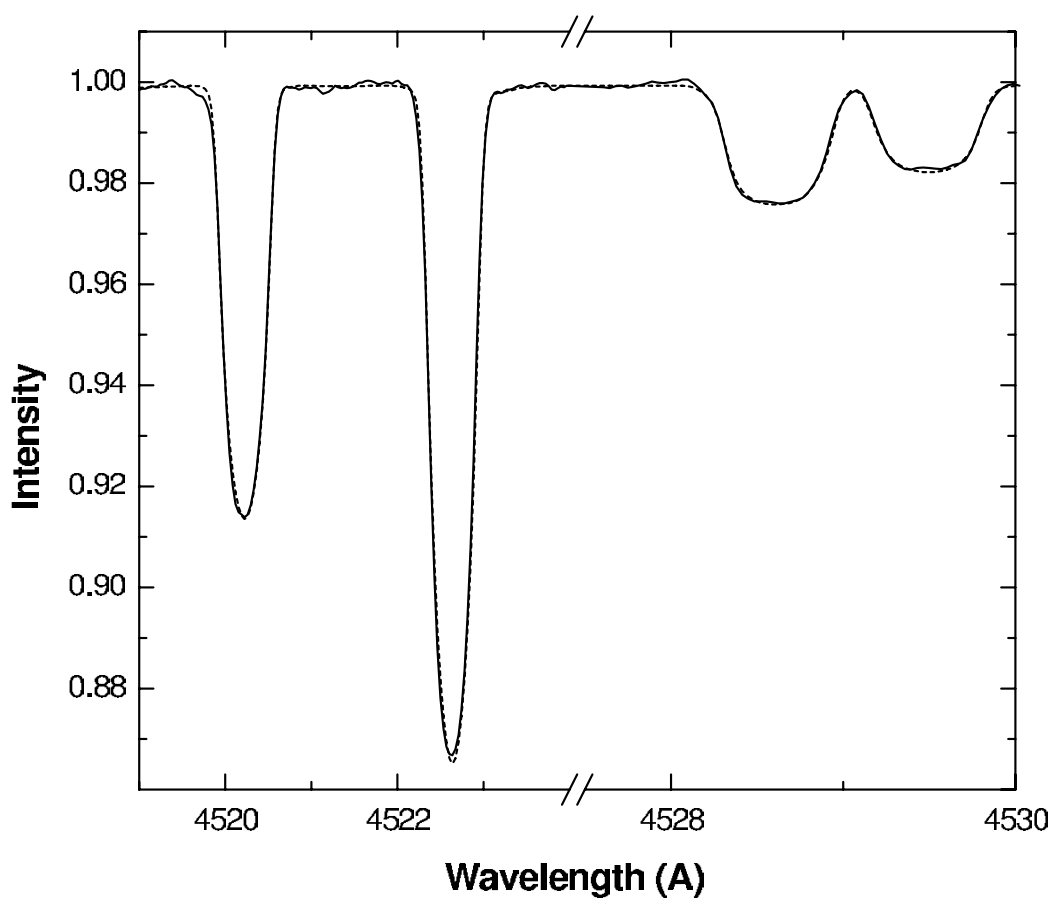

Figure 4. Vega spectrum showing the synthetic spectrum (dashed line) and the observed line profiles (solid line) for the $\lambda 4519-4530$ region. The model parameters are $V \sin i=21.9 \mathrm{~km} \mathrm{~s}^{-1}$, polar $T_{\mathrm{eff}}=9600 \mathrm{~K}$, polar $\log g=3.94 \mathrm{dex}, V_{\mathrm{eq}}=160 \mathrm{~km} \mathrm{~s}^{-1}, \xi_{\mathrm{T}}=1.07 \mathrm{~km} \mathrm{~s}^{-1}$ and $i=7.7^{\circ}$.

of the weak lines. The fit to the $\lambda 4528-4530$ region is given in Table 1 and has an $\mathrm{rms} \sim 1.3 \times 10^{-6}$ about one half of the expected $S / N \sim 2000$. The results are illustrated in Figure 4 . The reader should take note that the $T_{\text {eff }}$ and $\log g$ of this model are not the final values but have been decreased to allow for the abundance and $\log g f$ errors surmised above.

As in our earlier study we tested the uniqueness of the solution by generating extensive grids to explore the multidimensional surface in detail and we are confident that our solution is unique. Thus the five parameters for Vega are polar $T_{\text {eff }}=9680 \pm 3 \mathrm{~K}$, polar $\log g=4.00 \pm 0.01 \operatorname{dex}, V \sin i=21.9 \pm 0.1 \mathrm{~km} \mathrm{~s}^{-1}, \xi=1.09 \pm 0.01 \mathrm{~km} \mathrm{~s}^{-1}$ and $\omega / \omega_{c}=0.47 \pm 0.01 . T_{\text {eff }}$ and $\log g$ come from the iterations between the continuous energy flux and the hydrogen-line profiles and the other parameters from the line analysis. The breakup fraction implies an inclination $i=7.8 \pm 0.1^{\circ}$. The value of $\xi_{\mathrm{T}}=1.1 \mathrm{~km} \mathrm{~s}^{-1}$ can be compared to that of $0.6 \mathrm{~km} \mathrm{~s}^{-1}$ from Adelman \& Gulliver (1990) using classical techniques or $1.0 \mathrm{~km} \mathrm{~s}^{-1}$ of Hill \& Landstreet (1993) using a spectrum fitting technique. The derived mean $\log g$ value of 3.97 is close to the value normally used to model Vega $\left(\log g=3.95\right.$, Castelli \& Kurucz 1994) and the mean $T_{\text {eff }}=9506 \pm 10 \mathrm{~K}$ is within the errors of Ciardi et al. 's (2001) direct determination of $T_{\text {eff }}=9555 \pm 111 \mathrm{~K}$.

\section{Conclusions}

It is unfortunate that the premiere photometric standard upon which our spectrophotometry is anchored should be the object it is - a rapidly-rotating star seen almost pole-on and surrounded by a ring of dust - but this circumstance need not be an impediment to its use as a standard. It simply means that the fitting model should be more complex 
than a spherical one and in any case is far more testing of the models than a simple spherical comparison.

\section{Acknowledgements}

SJA and AFG thank Dr. James E. Hesser, Director of the Dominion Astrophysical Observatory for the observing time. Financial support was provided to AFG by the Natural Sciences and Engineering Research Council of Canada. SJA's contribution to this paper was supported in part by grants from The Citadel Foundation.

\section{References}

Adelman, S.J. 1988, MNRAS, 230, 671

Adelman, S.J., \& Gulliver, A.F. 1990, ApJ, 348, 712

Adelman, S. J., Gulliver, A. F., \& Heaton, R. J. 2004, submitted to A\&A

Aumann, H. H. et al. 1984, ApJ, 96, L23

Bohlin, R.C., Harris, A.W., Holm, A.V., \& Gry, C. 1990, ApJS, 73, 413

Bohlin, R.C., Dickinson, M.E. \& Calzetti, D. 2001, AJ, 122, 2118

Castelli, F., \& Kurucz, R.L. 1994, A\&A, 281, 817

Ciardi, D.R., van Belle, G.T., Akeson, R.L., Thompson, R.R., \& Lada, E.A., 2001, ApJ, 559, 1147

ESA, 1997, The Hipparcos and Tycho Catalogs, SP-1200

Gray, R.O. 1985, JRASC, 79, 237

Gray, R.O. 1988, JRASC, 82, 336

Gulliver, A.F., Hill, G., \& Adelman, S.J. 1994, ApJ, 429, L81

Hanbury Brown,R., Davis, J., \& Allen, L.R. 1974, MNRAS, 167, 121

Hayes, D.S. 1985, in "Calibration of Fundamental Stellar Quantities", eds. D. S. Hayes, L.E. Pasinetti, \& A.G.D. Philip, (Dordrecht: Reidel), 225

Heinrichsen, I., Walker, H.J., \& Klaas, U. 1998, MNRAS, 293, L78

Hill, G. 1979, Publ. Dominion Astrophysical Obs., 15, 279

Hill, G.M., \& Landstreet, J.D. 1993, A\&A, 276, 142

Johnson, H.L., \& Morgan, W.W. 1953, ApJ, 117, 313

Kurucz, R.L. 1993, ASP Conference Series, 44, 87 (see also CD-ROM distribution)

Kurucz, R.L., \& Avrett, E.H. 1981, SAOSpecRep, 391

Peterson, D.M. 1969, SAOSpecRep, 293

Peterson, D.M. 2003, private communication

van der Bliek, N.A., Prusti, T., \& Waters, L.B.F.M. 1994, A\&A, 285, 229

\section{Discussion}

E. GRIFFIN: Do you remove the IS components from your observed spectra?

S. Adelman: We will have to do this for modelling these lines with them. But for the lines we have analyzed so far this is not necessary. For our in process atlases we will leave the IS components in the data. 